\title{
The Explaining of the Elementary Particles Cold Genesis by a Preonic Quasi-Crystal Model of Quarks and a Pre-Quantum Theory of Fields
}

\author{
Marius Arghirescu \\ State Office for Inventions and Trademarks, Bucharest, Romania \\ Email address: \\ arghirescu.marius@osim.ro,maris3a@yahoo.com \\ To cite this article: \\ Marius Arghirescu. The Explaining of the Elementary Particles Cold Genesis by a Preonic Quasi-Crystal Model of Quarks and a \\ Pre-Quantum Theory of Fields. International Journal of High Energy Physics. Vol. 5, No. 1, 2018, pp. 12-22. \\ doi: $10.11648 /$ j.ijhep.20180501.13
}

Received: March 2, 2018; Accepted: March 16, 2018; Published: April 9, 2018

\begin{abstract}
Through a preonic quasi-crystalline quark model, resulted as Bose-Einstein condensate of gammons: $\gamma^{*}=\left(\mathrm{e}^{+} \mathrm{e}^{-}\right)$ and by a pre-quantum cold genesis theory of matter and fields, which predicted the existence of a preon $\mathrm{z}^{0} \approx 34 \mathrm{~m}_{\mathrm{e}}$ experimentally evidenced in 2015, the elementary particles genesis is explained by the cold genesis of two preonic bosons with hexagonal symmetry: $z_{\pi}=7 z^{0} ; z_{2}=4 z^{0}$, which explains also the stability of quarks, by a mechanism with a first step of $z^{*} /\left(q^{ \pm}\right.$ $\left./ \mathrm{q}^{0}\right)^{*}$ - pre-cluster forming by magnetic interaction and a second step of $\mathrm{z} /\left(\mathrm{q}^{ \pm} / \mathrm{q}^{0}\right)$ - collapsed cluster forming, with the aid of magnetic confinement, with $\mathrm{z}=\left(\mathrm{z}^{0}, \mathrm{z}_{2}, \mathrm{z}_{\pi}\right)$ and $\left(\mathrm{q}^{ \pm} / \mathrm{q}^{0}\right)$ - quark or pseudo-quark, resulting some predictions for bosonic dark matter constituents and for multi-quark particles of cold genesis, such as: $2450 \mathrm{~m}_{\mathrm{e}} ; 2685.4 \mathrm{~m}_{\mathrm{e}}$ tetra-quark; $3063.8 \mathrm{~m}_{\mathrm{e}}$ pentaquark; $2720 \mathrm{~m}_{\mathrm{e}}, 3672.4 \mathrm{~m}_{\mathrm{e}}$ hexa-quark; $3329 \mathrm{~m}_{\mathrm{e}}, 4762.2 \mathrm{~m}_{\mathrm{e}}$ hepta-quark.
\end{abstract}

Keywords: Preonic Quark, Quasi-Crystal Quark, Bose-Einstein Condensate, Particles Cold Genesis, Dark Matter, Gammon

\section{Introduction}

In a theory developed by author in the book: "The cold Genesis of Matter and Fields" (Ed. SciencePG, 2015, [1-4]), is argued the possibility of a cold genesis of elementary particles in a very strong magnetic field, comparable to those of a magnetar or gravistar, in accordance with a resulted quasi-crystalline model of quark and particle, resulted as Bose-Einstein condensate of $\mathrm{N}$ gammons, considered as pairs $\gamma^{*}\left(e^{*+}-e^{*-}\right)$ of quasi-electrons with diminished mass $\mathrm{m}_{\mathrm{e}}{ }^{*}$, charge $\mathrm{e}^{*}=\left({ }^{2} / 3\right)$ e and magnetic moment $\mu_{\mathrm{e}}{ }^{*}$, whose etheronoquantonic vortex of the magnetic moment: $\Gamma_{\mu}^{*}(\mathrm{r})=\Gamma_{\mathrm{A}}+\Gamma_{\mathrm{B}}$,

$$
F_{s}(r)=q_{s} \cdot E_{s} ; \quad E_{s}(r)=k_{1} \cdot \rho_{e}(r) \cdot v_{\mathrm{c}}^{2}=\frac{1}{2} k_{1} \cdot \frac{\Delta p_{c}}{\Delta t} ;
$$

- The resulted quark/particle model explains the nuclear force as an attraction of the nucleon's impenetrable volume $v_{i}$ in the field of $\mathrm{N}^{\mathrm{p}}=(2 \mathrm{~N}+1)$ - superposed vortices $\Gamma_{\mu}^{*}(\mathrm{r})$ of another nucleon, having an exponential variation of quanta impulse density: $p_{\mu}=\rho_{\mu} c$, according to equation: is formed of sinergonic etherons $\left(\mathrm{m}_{\mathrm{s}} \approx 10^{-60} \mathrm{~kg}\right)$ - generating the magnetic potential $\mathrm{A}$ and of quantons $\left(\mathrm{m}_{\mathrm{h}}=\mathrm{h} / \mathrm{c}^{2}=\right.$ $\left.7.37 \times 10^{-51} \mathrm{~kg}\right)$ - generating vortex-tubes that materializes the B-field lines of magnetic induction.

- The used electron model is with the charge: e $=\mathrm{S}^{0} / \mathrm{k}_{1}$ contained by its surface $\mathrm{S}^{0}=4 \pi \mathrm{a}^{2}$ of radius: $\mathrm{a}=1.41 \mathrm{fm}-$ close to the value of the nucleon radius resulted from the expression of the nuclear volume: $r_{n} \approx 1.25 \div 1.5 \mathrm{fm}$, and with an exponential variation of its density: $\rho_{e}=\rho_{e}{ }^{0} e^{-r / \eta}$; with: $\rho_{e}{ }^{0}$ $=22.24 \mathrm{~kg} / \mathrm{m}^{3}$ and $\eta=0.965 \mathrm{fm},\left(\rho_{e}(a)=5.17 \times 10^{13} \mathrm{~kg} / \mathrm{m}^{3}\right)$.

The electric field intensitiy E, the electric charge $\mathrm{q}$ and the magnetic induction $\mathrm{B}$ the results in the form:

$\mathrm{q}_{\mathrm{s}}=\frac{4 \pi \cdot \mathrm{r}_{\mathrm{q}}^{2}}{\mathrm{k}_{\mathrm{l}}} ; \quad \mathrm{B}=\mathrm{k}_{1} \cdot \rho_{\mu}(r) \cdot \mathrm{v}_{\mathrm{c}} ; \quad\left(k_{1}=\frac{4 \pi \cdot a^{2}}{e} ; \mathrm{v}_{\mathrm{c}} \approx c\right)$

$$
V_{n}(r)=v_{i} P_{n}=V_{n}^{0} \cdot e^{-r / \eta^{*}} ; V_{n}{ }^{0}=v_{i} P_{n}{ }^{0} ; P_{n}(r)=(1 / 2 /) \rho_{n}(r) \cdot c^{2}
$$

with: $v_{\mathrm{i}}(0.6 \mathrm{fm}) \approx 0.9 \mathrm{fm}^{3}$ - the impenetrable quantum volume of the nucleon; $\rho_{n}^{0} \approx N^{p} \cdot \rho_{e}^{0}$, and with $\eta^{*}=0.755 \mathrm{fm}$, resulted by the condition: $\rho_{\mu}\left(a, \mathrm{e}^{*}\right) \approx \rho_{e}\left(a, \mathrm{e}^{*}\right)=\left({ }^{2} / 3\right) \rho_{e}(a, \mathrm{e}) \sim \sim$ 
$\mathrm{e}^{*}=(2 / 3) \mathrm{e}$, (equal with the up-quark charge), [1-4].

- The theory- which predicted the existence of a preon $\mathrm{z}^{0} \approx$

$34 \mathrm{~m}_{\mathfrak{e}}$, experimentally evidenced in 2015 but considered as $\mathrm{X}$ - boson of a fifth force, of leptons to quark binding, argues a preonic model of quarks whose stability was explained by a quasi-crystalline model of $\mathrm{z}^{0}$-preon and of the quark core.

- The virtual radius: $r_{\mu}{ }^{n}$ of the proton's magnetic moment, $\mu_{\mathrm{p}}$, results -in the theory, by a degenerate Compton radius: $r_{\lambda}$ $=\lambda / 2 \pi=h / m_{p} c$ of an attached positron included in the $\mathrm{N}^{\mathrm{p}}$ cluster volume with $\mathrm{N}^{\mathrm{p}} \approx 2 \mathrm{~N}$ quasi-electrons, by its decreasing from the value: $r_{\mu}^{e}=3.86 \times 10^{-13} \mathrm{~m}$, to the value: $r_{i}$ $=r_{\mu}^{p}=0,59 f m$, as a consequence of the increasing of the impenetrable quantum volume mean density in which is included the protonic positron centroid: $\mathrm{m}_{0}$, from the value: $\bar{\rho}_{\mathrm{e}}$ to the value: $\overline{\rho_{n}} \cong f_{d} \cdot N^{p} \cdot \overline{\rho_{e}}$, conform to the equation:

$$
\mu_{p}=k_{p} \frac{m_{e}}{m_{p}} \mu_{e}=k_{p} \frac{\overline{\rho_{e}}}{\rho_{n}} \mu_{e} \cong k_{p} \frac{1}{f_{d} \cdot N^{p}} \mu_{B p}=\frac{e \cdot c \cdot r_{\mu}^{p}}{2} ; \quad k_{p}=\frac{g_{p}}{g_{e}}=2.79 ; k_{p}=\frac{\rho_{n}\left(r^{+}\right)}{\rho_{n}^{0}}=e^{-\frac{r^{+}}{\eta_{d}}}
$$

in which: $\mathrm{k}_{\mathrm{p}}$-the gyromagnetic ratio; $\bar{\rho}_{\mathrm{e}} ; \bar{\rho}_{\mathrm{n}}$ - the mean density of electron and nucleon; $r^{+}$-the position of protonic positron centrol in report with the proton centre; $f_{d}$-the degeneration coefficient of the quasielectron mass, $\mathrm{m}_{\mathrm{e}}{ }^{*},\left(\mathrm{f}_{\mathrm{d}} \approx\right.$ 0.81 ). The theory gives: $r_{e}^{+}=0.96 \mathrm{fm}$ for the protonic positron axially positioned inside the protonic quantum volume, [2-4].

- The neutron results in CGT by a specific "dynamid" model, with a degenerate electron with degenerate magnetic moment: $\mu_{\mathrm{e}}^{\mathrm{s}}=-4.597 \mu_{\mathrm{N}}$ rotated inside the quantum volume of a proton by the etherono-quantonic vortex $\Gamma \mu_{p}$ of its magnetic moment $\mu_{\mathrm{p}}$, with a speed $\mathrm{v}_{\mathrm{e}} \approx 1.7 \times 10^{-2} \mathrm{c}$, to an orbital with a radius: $r_{e}{ }^{*} \approx 1.283 \mathrm{fm}$ under dynamic equilibrium of forces on tangent and radial directions, [3]. The difference between the neutron's mass and the proton's mass: $\Delta_{\mathrm{n}} \approx 2.6 \mathrm{~m}_{\mathrm{e}}$, is given by a "weson" $\mathrm{w}^{-}$composed of the attached negatron and a linking gammon: $\sigma\left(\mathrm{e}^{*+}-\mathrm{e}^{*}\right)$ which is transformed into an antineutrino given as pair of electronic centroids, by the loss of the quantum volume of quasielectrons, the quasielectron mass resulting of value:

$$
\mathrm{m}_{\mathrm{e}}^{*}=\left(\Delta_{\mathrm{n}}-1\right) / 2 \approx 0.81 \mathrm{~m}_{\mathrm{e}}[4,5] .
$$

$$
{ }^{0} n_{\mathrm{e}}=\left(\mathrm{N}^{\mathrm{p}}+\mathrm{w}^{-}\right)=\left(\mathrm{N}^{\mathrm{p}}+\mathrm{e}^{-}+\sigma\right) \rightarrow{ }^{1} \mathrm{p}_{\mathrm{r}}+\mathrm{e}^{-}+\overline{\mathrm{v}}_{\mathrm{e}}+\epsilon_{\sigma}(889 \mathrm{keV}) ;
$$

- The quarks confinement force is explained in CGT by a "bag" model given by the static quantum pressure gradient of the scalar charge of impenetrable quantum volume of the nucleon, [6], which explains also its repulsive property, observed in scattering interactions.

\section{The Preonic Quasi-Crystal Quark Model}

- The necessity of the preon with $\sim 34 \mathrm{~m}_{\mathrm{e}}$ resulted in CGT by the conclusion that -in a cold genesis scenario, of 'vortexes cascade' particles forming process, their masses are given according to the sum rule as integer number of basic preons $z^{0}$ of linked quarks. As helpful theory for this issue was used a theoretical result of Olavi Hellman which deduces the value of elementary particles mass, by a simplified relation:

$$
\mathrm{M}_{\mathrm{p}}=\left(\mathrm{K}_{\mathrm{m}} / 2 \alpha\right) \mathrm{m}_{\mathrm{e}},\left(\alpha=\mathrm{e}^{2} / \hbar \mathrm{c}=1 / 137\right)
$$

with a tolerance under $1 \%$, neglecting the electromagnetic field contribution, by integer values of $\mathrm{K}_{\mathrm{m}}$, as a multiple of the mass: $\mathrm{M}_{0}=68.5 \mathrm{~m}_{\mathrm{e}} ;\left(\mathrm{K}_{\mathrm{m}}=3 ; 4 ; 14\right.$ for the mesons $\mu, \pi, \mathrm{K}$ and $\mathrm{K}_{\mathrm{m}}^{\mathrm{p}}=27$ for the proton' mass), but which not gives an enough satisfying value for the proton mass, (i.e $1849.5 \mathrm{~m}_{\mathrm{e}}$ instead 1836,1 $\mathrm{m}_{\mathrm{e}}$ ).

Re-gauging the mass of the basic preon with the proton mass and the $\Sigma$-particle mass, it resulted as plausible the preonic mass value: $z^{0}=34 \mathrm{~m}_{\mathrm{e}}$, imposing a tolerance of \pm 0.5 $\mathrm{m}_{\mathrm{e}}$ for the resulted proton' mass, i.e:

$$
\mathrm{z}^{0}=\left(\mathrm{m}_{\mathrm{pr}} \pm 0.5 \mathrm{~m}_{\mathrm{e}}\right) /\left(\mathrm{n} \cdot \mathrm{K}_{\mathrm{m}}{ }^{\mathrm{p}}\right),
$$

with $\mathrm{n}=2$-given by the mass of the $\Sigma$-particle, $(\sim 2340$ $\mathrm{m}_{\mathrm{e}}$ ). - By CGT was argued the fact that- in a cold genesis model, the preon $\mathrm{z}^{0}$ may results as cluster of $\mathrm{n}=42$ degenerate electrons with the mass: $\mathrm{m}_{\mathrm{e}}{ }^{*} \approx 0.81 \mathrm{~m}_{\mathrm{e}}$ and with the super-dense kernels (centroids) vortexially confined in a volume of radius $r_{z}<0.2 \mathrm{fm}$, the $\mathrm{z}^{0}$ cluster being given as a pair of quarcins: $\mathrm{c}^{* \pm}=21 \mathrm{~m}_{\mathrm{e}}^{*}$, with hexagonal symmetry $(7 \times 3=21$ quasielectrons- figure $1, \mathrm{e})$, which decay in the form:

$$
\mathrm{z}^{0}\left(\mathrm{c}_{0}{ }^{*}+\overline{\mathrm{c}}_{0}^{*}\right) \rightarrow \mathrm{c}_{0}^{+}+\mathrm{c}_{0}^{-} \rightarrow \mathrm{e}^{+}+\mathrm{e}-;
$$

The stability of the quarcin $\mathrm{c}^{* \pm}=21 \mathrm{~m}_{\mathrm{e}}{ }^{*}$ is explainedaccording to the model, by its quasi-crystallin arrangement of electronic centroids of the kernel and by its quasi-equality between its diameter and its length (giving its virtual framing into a sphere).

The resulted sub-structure of the fundamental elementary particles, considered as formed "at cold", by quarks with effective mass (giving the particle's mass by the sum rule) and fractional electric charge: $\mathrm{q}^{*}=\left(+2 /{ }_{3} \mathrm{e} ;-1 /{ }_{3} \mathrm{e}\right)$, formed as preonic clusters, is given by the preon $\mathrm{z}^{0}$ considering also the existence of the zerons:

$$
\begin{gathered}
\mathrm{z}_{1}=3 \mathrm{z}^{0}=102 \mathrm{~m}_{\mathrm{e}} ; \mathrm{z}_{2}=4 \mathrm{z}^{0}=136 \mathrm{~m}_{\mathrm{e}} ; \\
\mathrm{z}_{\pi}=\left(\mathrm{z}_{1}+\mathrm{z}_{2}\right)=7 \mathrm{z}^{0}=238 \mathrm{~m}_{\mathrm{e}},
\end{gathered}
$$

with the following sub-structures $[1-3,5]$ : 
Table 1. Elementary particles: (theoretic mass) / (experimentally determined mass).

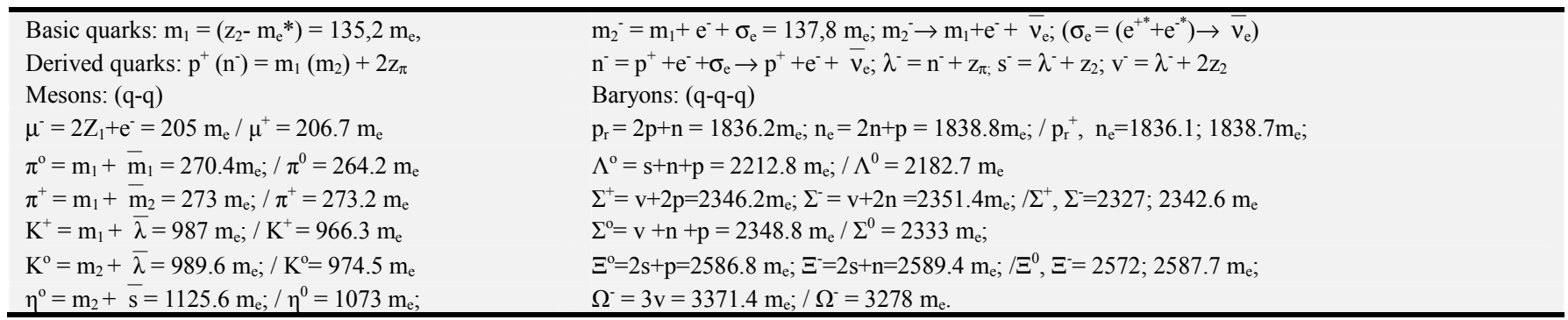

The quarks with negative charge $\left({ }^{-1} / 3\right) \cdot e: m_{2}^{-}, n^{-}, \lambda^{-}, s^{-}, v^{-}$, results in CGT as cold lepto-quarks, i.e- formed by a cold quark with positive charge $\left(+^{2} / 3\right) \cdot e: \mathrm{m}_{1}^{+}, \mathrm{p}^{+}$and an attached electron with degenerate magnetic moment or also a $\mathrm{z}_{\pi^{-}}$zeron $\left(\rightarrow \lambda^{-}\right)$and one or two $\mathrm{z}_{2}-$ zeron $\left(\rightarrow \mathrm{s}^{-}, \mathrm{v}^{-}\right)$.

According to the model, the masses of the elementary particles (table 1) results by a quasicrystallin form of quarks with hexagonal simmetry, given by the simmetry of the considered $z^{0}$ - preon and of the $z_{\pi}=7 z^{0}$ and $z_{2}=4 z^{0}$ zerons, like in figure 1 , and by a quark equation:

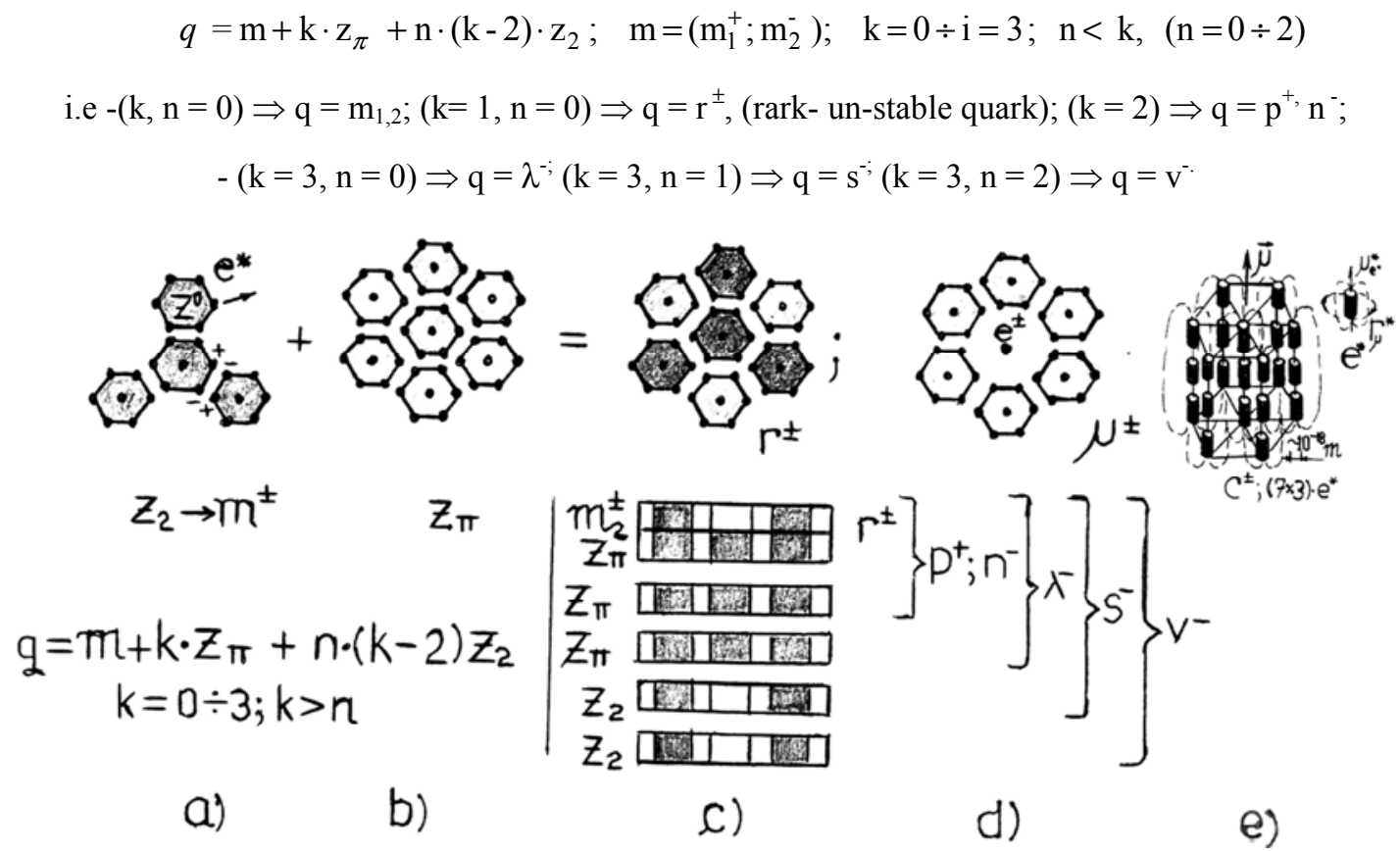

Figure 1. a) m- basic quark; b) quasi-crystallin form of $z_{\pi}$; c) quasi-crystallin structure of quarks, (CGT). d) quasi-crystallin ring form of $\mu^{ \pm}$; e) the quasicrystallin form of the quarcin $c^{ \pm}=21 \mathrm{~m}_{e}{ }^{*}$.

From eqn. (9), the baryons mass results as combinations (q-q-q), according to the equation:

$$
M_{b}=\mathrm{M}_{\mathrm{q}}+\mathrm{k} \cdot \mathrm{z}_{\pi}+\mathrm{n} \cdot(\mathrm{k}-6) \cdot \mathrm{z}_{2} ; \mathrm{M}_{\mathrm{q}}=\sum_{\mathrm{i}=1}^{3} \mathrm{~m}_{\mathrm{i}} ; \mathrm{m}_{\mathrm{i}}=\left(\mathrm{m}_{1}^{+} ; \mathrm{m}_{2}^{-}\right) ; \mathrm{k}=6 \div 9 ; \mathrm{n} \leq 2
$$

Some "resonance" particles (*) may be formed also "at cold":

$$
\Delta^{\mathrm{o}^{*}}=2 \mathrm{v}+\mathrm{p}=2858.8 \mathrm{~m}_{\mathrm{e}} ; \Delta^{-*}=2 \mathrm{v}+\mathrm{n}=2861.4 \mathrm{~m}_{\mathrm{e}} ;
$$

and: $\Xi^{-*}=3 \mathrm{~s}^{-}=2963.4 \mathrm{~m}_{\mathrm{e}}$; (known mass: $2850 \mathrm{~m}_{\mathrm{e}} ; 3004 \mathrm{~m}_{\mathrm{e}}$ ).

The theory predicts also the existence of the next particles:

$$
\Phi^{-}=2 \mathrm{v}+\lambda=3099.4 \mathrm{~m}_{\mathrm{e}} ; \Phi^{-*}=2 \mathrm{v}+\mathrm{s}=3235.4 \mathrm{~m}_{\mathrm{e}} ;
$$

$2 \mathrm{~s}+\lambda=2827.4 ; 3 \mathrm{~s}=2963.4 \mathrm{~m}_{\mathrm{e}} ; \Lambda^{+}=\mathrm{s}+2 \mathrm{p}=2210.2 \mathrm{~m}_{\mathrm{e}}$;

$$
\Lambda^{-}=\mathrm{s}+2 \mathrm{n}=2215.4 \mathrm{~m}_{\mathrm{e}} ; \mathrm{Z}_{\pi}=\left(\mathrm{Z}_{1}+\mathrm{Z}_{2}\right)=238 \mathrm{~m}_{\mathrm{e}} ;
$$

The structure of a quark with effective mass which gives the particle's mass according to the sum rule, results in CGT by a current quark mass, of $6 \div 10 \mathrm{MeV} / \mathrm{c}^{2}$, given by its impenetrable quantum volume: $v_{q i}\left(r_{p}\right)$ and the maximal density of the particle's kernel, [6] and a quantum volume $v_{\mathrm{qv}}$ of confined vectorial photons (vexons), which forms- in CGT, the vortexial equivalent of the gluonic shell considered in Quantum Chromodynamics.

The current quark mass has -in the model, a scalar shell of 
$\sim 0.1 \mathrm{fm}$ thickness, which envelops the current mass of quark and which may explain- according to CGT, the value of $\sim 0.3$ $\mathrm{fm}$ for the quark radius determined by some scattering experiments as those made at Fermilab, [7], evidenced for proton by $p$-p scattering at few $\mathrm{TeV}$ [8], which may be considered also for the quark by similitude with the repulsive scalar shell of the protonic impenetrable quantum volume and explains their mutual interaction, [6].

\section{The Mechanism of Preonic Cold Quarks Forming and of Dark Matter Bosons Forming}

Because that the basic quark: $m_{1}$ results (according to the model) from a quasi-crystallin 'zeron' $z_{2}$ (neutral

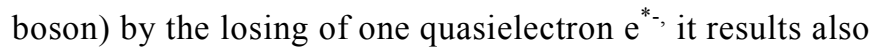
the possibility of neutral pseudo-quarks cold forming (bosonic pseudo-quarks), and thereafter- of neutral 'dark' bosonic particles, which- at low temperature $\mathrm{T} \rightarrow 0 \mathrm{~K}$, i.e mainly in the interstallary but also in the interplanetary space, are not radiative particles and may constitute dark matter particles. The possibility of the preonic cold quarks and pseudo-quarks forming results as consequence of the next forces:

a) Because that it may be considered a quantum impenetrable volume $v_{\mathrm{q}}$ not only for baryons but also for mesons and quarks [5], it results that also the $z^{0}$ - preons and even the electrons have a residual impenetrable volume, $v_{i}{ }^{e}$ by which they may interact by a residual 'strong' force $\mathrm{F}_{\mathrm{qn}}=-\nabla \mathrm{V}_{\mathrm{n}}$ (d) resulted from a potential conform to eqn. (2), with the value of $v_{i}$ approximated by an (semi) empiric relation [5]:.

$$
\vartheta_{k i}=\vartheta_{n i} \cdot e^{-K\left(1-\frac{m_{k}}{m_{p}} \cdot k\right)} ; \mathrm{k}=e^{\left(1-\frac{m_{k}}{m_{p}}\right)} ; \mathrm{K}=8.97 ; \vartheta_{n i}\left(\mathrm{~m}_{\mathrm{p}}\right) \approx 0.9 \mathrm{fm}^{3} ; \mathrm{m}_{\mathrm{p}} \approx 1836 m_{e}
$$

in which the term ' $\mathrm{k}$ ' take into account the fact that- inside the quantum volume of a bigger particle, the value $v_{\mathrm{ki}}$ of a smaller particle increases proportional with the local density, as in the case of quarks.

For (quasi) free $m_{k}$ particles or particles with $m_{k}>m_{p}$, we have: $\mathrm{k}=1$. It results that- for: $\mathrm{m}_{\mathrm{k}}=\mathrm{m}_{\mathrm{z}}{ }^{0}=34 \mathrm{~m}_{\mathrm{e}} \Rightarrow \mathrm{v}_{\mathrm{zi}} \approx$ $1.77 \times 10^{-4} \mathrm{fm}^{3}, \mathrm{r}_{\mathrm{zi}} \approx 3.48 \times 10^{-17} \mathrm{~m}$, inside a bigger particle and: $v_{\mathrm{zi}} \approx 1.35 \times 10^{-4} \mathrm{fm}^{3}, \mathrm{r}_{\mathrm{zi}} \approx 3.2 \times 10^{-17} \mathrm{~m}$, in the free state, so we may conclude that the kernel of the preonic $\mathrm{z}^{0}$-cluster of 42

electronic superdense centroids with mass $\mathrm{m}_{0} \approx 5 \times 10^{-5} \mathrm{~m}_{\mathrm{e}}$ and radius $\mathrm{r}_{0} \approx 10^{-18} \mathrm{~m}$, has the radius: $\mathrm{r}_{\mathrm{zi}} \approx 3 \times 10^{-17} \mathrm{~m},[5]$,

b) Analyzing the way in which the $z^{0}$-preons are formed, we may conclude by CGT that the gammonic pairs of quasielectrons: $\gamma^{*}\left(\mathrm{e}^{*+}-\mathrm{e}^{*-}\right)$ have the quasi-electrons coupled axially and they interact thereafter also radially, magnetically but also electrically, by a residual charge: $e_{r}{ }^{*}$ and magnetic moment: $\mu_{\mathrm{r}}{ }^{*}$, which- for $\mathrm{d} \leq 2 \mathrm{a}$, may be approximated in CGT [4] by the relations:

$$
\mathrm{e}_{\mathrm{r}}^{*}=\mathrm{f}_{\mathrm{v}} \cdot \mathrm{S}_{\mathrm{i}}^{*} / \mathrm{k}_{1},\left(\mathrm{k}_{1}=4 \pi \mathrm{a}^{2} / \mathrm{e} ; \mathrm{S}_{\mathrm{i}}^{*}=4 \pi \mathrm{r}_{\mathrm{i}}^{2}\right) ; \mathrm{r}_{\mathrm{i}}=\mathrm{d}_{\mathrm{i}} / 2 \leq \mathrm{a} \Rightarrow \mathrm{e}_{\mathrm{r}}^{*} / \mathrm{e}=\mathrm{f}_{\mathrm{v}} \cdot \mathrm{r}_{\mathrm{i}}^{2} / \mathrm{a}^{2 ;} \mu_{\mathrm{r}}^{*} \approx \mathrm{e}_{\mathrm{r}}^{*} \cdot \mathrm{r}_{\mathrm{i}} \cdot \mathrm{c} / 2
$$

in which $d_{i}$ represent the distance between the electronic centroids (between the interacting quasi-electrons) and $f_{v}\left(\varepsilon_{v}\right.$, $\left.\mathrm{T}_{\mathrm{c}}\right)<1$ is a proportionality factor, dependent on the vibration energy of the electronic kernel: $\varepsilon_{\mathrm{v}}=\mathrm{k}_{\mathrm{B}} \mathrm{T}_{\mathrm{c}}$, which diminish the values of the residual charge: $e_{r}{ }^{*}$ and magnetic moment: $\mu_{r}{ }^{*}$ by vectorial photons destruction.

The expression (12) of $\mathrm{e}_{\mathrm{r}}{ }^{*}$ and $\mu_{\mathrm{r}}{ }^{*}$ takes into account the fact that the radius $r_{i}$ of the surface $S_{i}^{*}$ which contains the residual charge cannot be bigger than the half of the interdistance $\mathrm{d}_{\mathrm{i}}$.

For a photon speed: $v_{\underline{i}} \sim \rho_{i}^{-1}$ inside the electron volume with the mean density $\bar{\rho}_{\mathrm{i}}$, the magnetic and the electric potential between two interacting quasielectrons have according to CGT [9], the expressions:

$$
\begin{gathered}
\mathrm{V}_{\mu \mathrm{i}}\left(\mathrm{d}_{\mathrm{i}}\right)=\mu_{\mathrm{r}}^{*} \cdot B_{i}(r)=\mu_{\mathrm{r}}^{*} \cdot k_{1} \rho_{i}\left(d_{i}\right) \cdot c \approx \frac{1}{f_{v}} \frac{e_{r}^{* 2}}{4 \pi \varepsilon_{0} \cdot d_{\mathrm{i}}} \mathrm{e}^{\frac{\mathrm{a}-\mathrm{d}_{\mathrm{i}}}{\eta}} ; \rho_{\mathrm{i}}\left(\mathrm{d}_{\mathrm{i}}\right)=\rho_{\mathrm{i}}^{0} \mathrm{e}^{\frac{\mathrm{d}_{\mathrm{i}}}{\eta}}=\rho(a) \cdot \mathrm{e}^{\frac{\mathrm{a}-\mathrm{d}_{\mathrm{i}}}{\eta}} ; \rho(a)=\frac{\mu_{0}}{k_{1}^{2}} ; \mu_{\mathrm{r}}^{*}=\frac{e_{\mathrm{r}}^{*} \cdot r_{i} \cdot c}{2} \\
\mathrm{~V}_{\mathrm{e}}\left(\mathrm{d}_{\mathrm{i}}\right) \approx \frac{e_{r}^{* 2}}{4 \pi \varepsilon_{0} \varepsilon_{r}^{i} \cdot d_{\mathrm{i}}} ; \frac{\mathrm{e}_{\mathrm{r}}^{*}}{\mathrm{e}}=\mathrm{f}_{\mathrm{v}} \frac{\mathrm{r}_{\mathrm{i}}^{2}}{\mathrm{a}^{2}} ; \quad \mathrm{r}_{\mathrm{i}}=\mathrm{d}_{\mathrm{i}} / 2 ; \quad \varepsilon_{\mathrm{r}}^{\mathrm{i}}=\varepsilon_{\mathrm{r}}\left(\rho_{\mathrm{i}}, \mathrm{d}_{\mathrm{i}}\right) ;
\end{gathered}
$$

It results that- if exists magnetic attraction but also electric repelling between two quasi-electrons with their quantum volume in mutual contact, neglecting the residual strong interaction between the quasi-electrons, the equilibrium distance $d_{i} \leq 2 \mathrm{a}$ of their interaction is given by the equilibrium equation:

$$
\mathrm{V}_{\mathrm{e}}\left(\mathrm{d}_{\mathrm{i}}\right)=\mathrm{V}_{\mu}\left(\mathrm{d}_{\mathrm{i}}\right) \Rightarrow \frac{e_{r}^{*_{2}}}{4 \pi \varepsilon_{0} \varepsilon_{r}^{i} \cdot d_{\mathrm{i}}}=\frac{1}{f_{v}} \frac{e_{r}^{*_{2}}}{4 \pi \varepsilon_{0} \cdot d_{\mathrm{i}}} \mathrm{e}^{\frac{\mathrm{a}-\mathrm{d}_{\mathrm{i}}}{\eta}} ; \Rightarrow \frac{1}{\mathrm{f}_{\mathrm{v}}} \varepsilon_{r}^{i} \cdot \mathrm{e}^{\frac{\mathrm{a}-\mathrm{d}_{\mathrm{i}}}{\eta}}=1 ; \quad \mathrm{r}_{\mathrm{i}}=\mathrm{d}_{\mathrm{i}} / 2 ; \varepsilon_{\mathrm{r}}^{\mathrm{i}}=\varepsilon_{\mathrm{r}}\left(\mathrm{d}_{\mathrm{i}}\right)
$$

Because that for $d_{i}>$ a it decreases also $\varepsilon_{\mathrm{r}}{ }^{i} \sim \rho_{\mathrm{i}}$, it results that the equality (15) is attained at the surface of the quantum volume of quasi-electron, i.e: at $\mathrm{d}_{\mathrm{i}}=\mathrm{a}$, if:

$$
\varepsilon_{\mathrm{r}}^{\mathrm{i}}(\mathrm{a}) / \mathrm{f}_{\mathrm{v}}(\mathrm{a})=1
$$

Considering that at the quasi-electron surface we have: 
$\varepsilon(\mathrm{a})=\varepsilon_{0}$ (the quantum vacuum electric permittivity), it results that: $\varepsilon_{\mathrm{r}}^{\mathrm{i}}(\mathrm{a})=1$ and that: $\mathrm{f}_{\mathrm{v}}(\mathrm{a})=1$. In this case we may approximate that the variation of the proportionality factor $f_{v}$ is according to the equation:

$$
f_{\mathrm{v}}=\mathrm{e}^{-\frac{\mathrm{a}-\mathrm{d}_{\mathrm{i}}}{\eta^{*}}} ; \eta^{*}=0.755 \mathrm{fm}
$$

Quasi-simultaneously, in the $\mathrm{z}^{0}$-cluster forming process, other three quasi-electrons with parallel residual magnetic moment but with opposed charge sign relative to the central quasi-electron are attracted magnetically by the first three quasi-electrons circularly disposed, forming a hexagonal precluster of quasi-electrons, (figure 2).

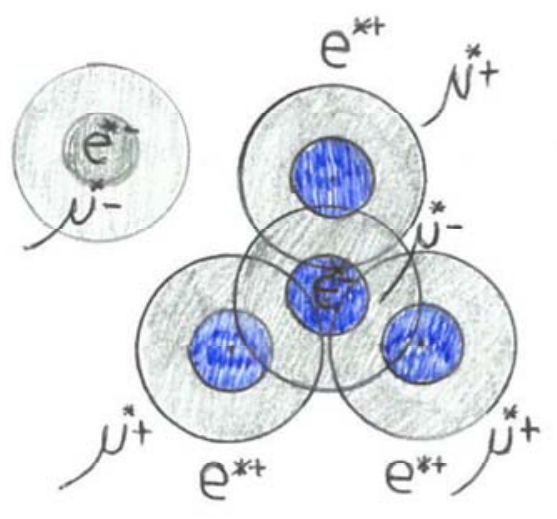

It results also that for $d_{i}<2 a$, the residual electric potential $\mathrm{V}_{\mathrm{e}}\left(\mathrm{d}_{\mathrm{i}}\right)$ decreases more rapidly than the residual magnetic potential $V_{\mu}\left(d_{i}\right)$ and it may be neglected at distances: $d_{i}<10^{-1}$ $\mathrm{fm}$, the residual magnetic potential $\mathrm{V}_{\mu}\left(\mathrm{d}_{\mathrm{i}}\right)$ generating the selfconfining of the pre-cluster formed by quasi-electrons with hexagonal or triangular symmetric arrangement, forming the cluster with radial magnetic attraction between the central quasi-electron and the other three quasi-electrons with antiparallel residual magnetic moment $\mu_{\mathrm{r}}{ }^{*}$ and with the same residual electric charge $\mathrm{e}_{\mathrm{r}}{ }^{*}$, (figure 2).
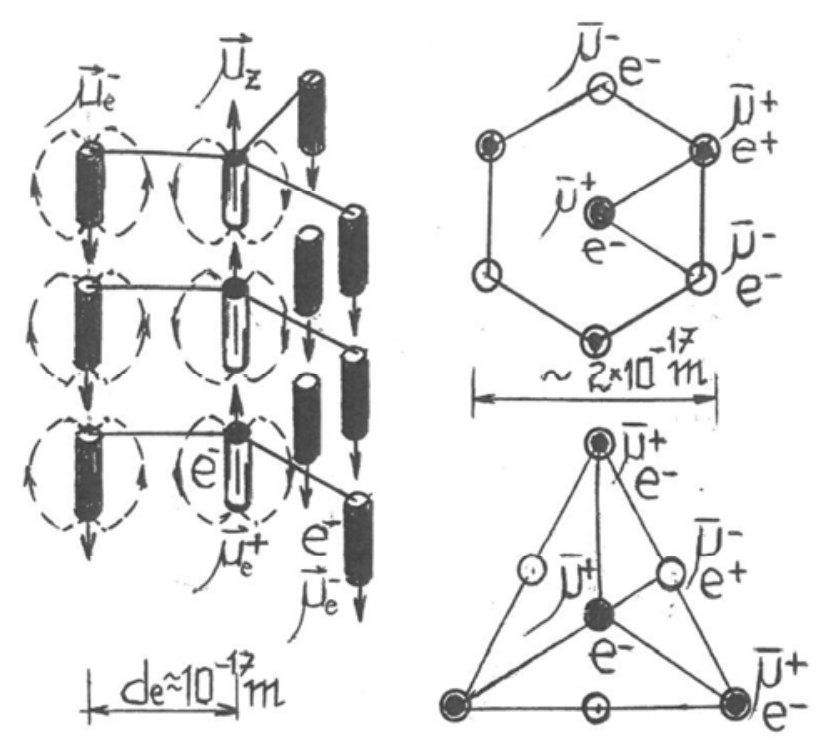

Figure 3. The forming of the $z^{0}$-cluster's kernel.

Simultaneously, at $\mathrm{T}_{\mathrm{c}} \leq \mathrm{T}_{\mathrm{BE}}\left(\mathrm{T}_{\mathrm{BE}}\right.$-the Bose-Einstein condensate forming temperature), the process is repeated with other quasielectrons arranged in a second level, axially coupled (magnetically) with the first seven quasielectrons, as their gammonic partner. In this way, by the repetition of the process, by magnetic self-confining, it may be formed a neutral $\mathrm{z}^{0}$-cluster, as Bose-Einstein condensate of $\mathrm{n}_{\mathrm{z}}=6 \mathrm{x} 7=$

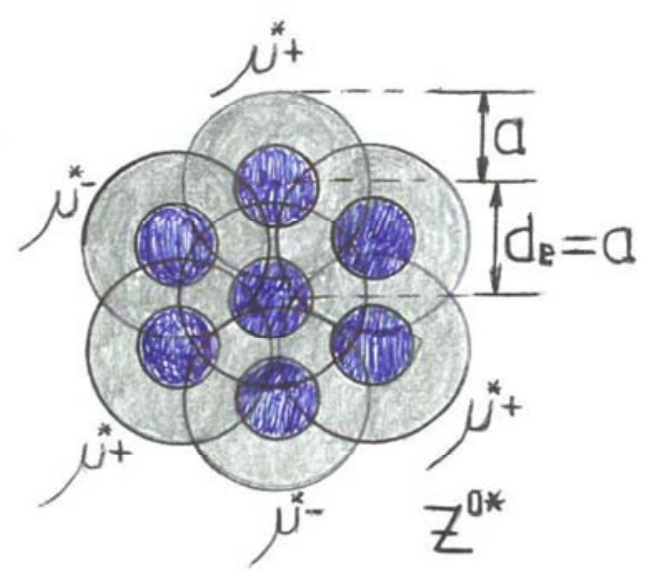

Figure 2. The $z^{0 *}$-pre-cluster forming.

$=42$ quasielectrons (figure 1, 3) with the electronic centroids approximated as being of barrel-like form with the radius: $r_{0}$ $\approx 10^{-18} \mathrm{~m}$ - according to some experiments of $\mathrm{x}$-rays scattering on electron [10] and axially aligned by axial magnetic attraction.

This $\mathrm{z}^{0}$-cluster may be considered as formed by two quarcins; $\mathrm{c}_{0}{ }^{+}, \mathrm{c}_{0}{ }^{-}$with mass $\mathrm{m}_{\mathrm{q}} \approx 17 \mathrm{~m}_{\mathrm{e}}$, charge: $\mathrm{e}^{*} \approx \pm\left({ }^{2} / 3\right) \cdot \mathrm{e}$ and with an own magnetic moment: $\mu_{\mathrm{q}}^{*}=\left(\mathrm{e}^{*} / \mathrm{m}_{\mathrm{q}}\right) \cdot \hbar / 2$.

Even if a specific low temperature $T_{c} \leq T_{B E}$ is generally enough for the forming of a neutral $\mathrm{z}^{0}$-cluster as bosonic $\mathrm{B}-\mathrm{E}$ condensate of gammons, for an increased probability of preons and of quarks cold forming is necessary a strong magnetic field, whose B-field quantonic vortex-tubes help the quasielectrons confining by an impulse density gradient which generates a confining magneto- gravitic force, [2-4].

It is observed also that- because the axial magnetic coupling of quarcins, the formed $\mathrm{z}^{0}$ - preon has also an own magnetic moment, $\mu_{\mathrm{z}}$, (as in the case of coupled atoms in a ferromagnetic material), by which it can interact magnetically with other preons forming a $\mathrm{z}_{2}$ boson ('zeron' in CGT) and thereafter -by magnetic attraction of other three $\mathrm{z}^{0}$-preons $-\mathrm{a} \mathrm{z}_{\pi}$ boson ('zeron'), which forms thereafter cold formed quarks by magnetic coupling with other $z_{\pi}$ - or /and $\mathrm{z}_{2}$-zerons and with a $\mathrm{z}_{2}$-zeron which becomes a basic $\mathrm{m}_{1}$ quark by the loosing of a quasi-negatron $\left(\mathrm{e}^{*}\right)$, like in the figure 1, a).

The cold formed quarks may be magnetically coupled not 
only radially, like in figure 4 , but also axially -orientation which increases the stability of the formed particle, as in the case of proton, we suppose, [6].

The preventing of quasielectrons cluster collapse at $\mathrm{T} \rightarrow 0 \mathrm{~K}$, may be explained by a repulsive force $\mathrm{F}_{\mathrm{rs}}$ of a scalar field generated by a remanent thermal vibration of the electronic centroids (kernels), by internal vectorial photons destruction, with a force variation approximated by eqn. (1), in the form:

$$
\mathrm{F}_{\mathrm{rs}}(\mathrm{d}, \mathrm{T})=\mathrm{q}_{0} \cdot \mathrm{E}_{\mathrm{r}}=-\nabla \mathrm{V}_{\mathrm{rs}}(\mathrm{d}) \approx \mathrm{q}_{0} \cdot \mathrm{k}_{1} \rho_{\mathrm{s}}(\mathrm{d}) \cdot \mathrm{c}^{2} ; \mathrm{q}_{0} \approx \frac{2 \pi \cdot \mathrm{r}_{\mathrm{e}}^{2}}{\mathrm{k}_{1}} ; \rho_{\mathrm{s}}(\mathrm{d})=\rho_{\mathrm{s}}^{0} \cdot \mathrm{e}^{\frac{-\mathrm{d}_{\mathrm{e}}}{\eta_{v}}} \propto \mathrm{k}_{\mathrm{B}} T_{c} ; \Rightarrow \mathrm{k}_{\mathrm{r}} \mathrm{q}_{\mathrm{v}}=\mathrm{k}_{1} \rho_{s}^{0} \cdot \mathrm{c}^{2}
$$

in which $\mathrm{q}^{0}$ is the static pseudo-charge of the repelled quasi-electron, given by the semi-surface of an (impenetrable) quantum volume: $v_{\mathrm{e}}\left(\mathrm{r}_{\mathrm{e}} \approx \mathrm{d}_{\mathrm{e}} / 2\right)$ and $\rho_{\mathrm{s}}(\mathrm{d})$ is the density of quanta (quantons and sinergonic etherons -according to CGT) released by the destroyed vectorial photons, which an spherical distribution and an intensity attenuation approximated as exponential, in accordance with a Boltzmann- type distribution of the released quanta with radial c- speed.

The magnetic force generated by the residual magnetic moment of the quasi-electrons, have the expressions:

$$
\mathrm{F}_{\mu}\left(\mathrm{d}_{\mathrm{i}}\right)=-\nabla \mathrm{V}_{\mu}\left(\mathrm{d}_{\mathrm{i}}\right)=-\frac{1}{\mathrm{f}_{\mathrm{v}}} \frac{e_{r}^{* 2}}{4 \pi \varepsilon_{0} \cdot d_{i}} \mathrm{e}^{\frac{\mathrm{a}-\mathrm{d}_{\mathrm{i}}}{\eta^{*}}}\left(\frac{1}{\mathrm{~d}_{\mathrm{i}}}-\frac{1}{\eta^{*}}\right) ; \mathrm{d}_{\mathrm{i}}<<\eta^{*}=0.755 \mathrm{fm}, \Rightarrow \mathrm{F}_{\mu}\left(\mathrm{d}_{\mathrm{i}}\right) \approx-\frac{1}{\mathrm{f}_{\mathrm{v}}} \frac{e_{r}^{* 2}}{4 \pi \varepsilon_{0} \cdot d_{i}^{2}} \mathrm{e}^{\frac{\mathrm{a}-\mathrm{d} i}{\eta^{*}}}=-\frac{e^{2} d_{i}^{2}}{64 \pi \varepsilon_{0} a^{4}}
$$

Because that inside the impenetrable volume $v_{i}{ }^{e}\left(r_{i}{ }^{0} \approx 0.03 \mathrm{fm}\right)$ of the quasi-electron the residual electric charge $e_{r}{ }^{*}$ becomes a pseudo-charge in the sense that ensures only the residual magnetic moment $\mu_{\mathrm{r}}{ }^{*}$, the electrostatic interaction at short interdistance $d_{i}<r_{i 0}$ may be neglected, the equilibrium inter-distance $d_{e}$ of the $z_{0}$-cluster of a quark, being given- according to eqs. (18) and (19), by the equality:

$$
\mathrm{F}_{\mathrm{rs}}\left(\mathrm{d}_{\mathrm{e}}\right)=\mathrm{F}_{\mu}\left(\mathrm{d}_{\mathrm{e}}\right) \Rightarrow \mathrm{q}_{0} \cdot \mathrm{k}_{1} \rho_{\mathrm{s}}\left(\mathrm{d}_{\mathrm{e}}\right) \cdot \mathrm{c}^{2}=\frac{\pi}{2} \cdot \mathrm{d}_{\mathrm{e}}^{2} \cdot \rho_{\mathrm{s}}\left(\mathrm{d}_{\mathrm{e}}\right) \cdot \mathrm{c}^{2}=\frac{e^{2} d_{e}^{2}}{64 \pi \varepsilon_{0} a^{4}} \Rightarrow \rho_{\mathrm{s}}\left(\mathrm{d}_{\mathrm{e}}\right)=\frac{\mu_{0}}{2 \cdot \mathrm{k}_{1}^{2}}=\frac{1}{2} \rho_{e}(\mathrm{a})=2.58 \times 10^{13} \mathrm{~kg} / \mathrm{m}^{3} ;
$$

Supposing an exponential variation of $\rho_{\mathrm{s}}(\mathrm{d})$ on a short distance, with the boundary condition: $\rho_{\mathrm{s}}(\mathrm{a}) / \rho_{\mathrm{s}}(0)<<1$, we may approximate in accordance with eq. (20), that:

$$
\rho_{\mathrm{s}}\left(\mathrm{d}_{\mathrm{e}}\right)=\frac{1}{2} \rho_{e}(\mathrm{a})=\rho_{\mathrm{s}}^{0} \cdot \mathrm{e}^{-\frac{\mathrm{d}_{\mathrm{e}}}{\eta_{\mathrm{v}}}}=2.58 \times 10^{13} \mathrm{~kg} / \mathrm{m}^{3} ; \eta_{v} \approx 10^{-1} \mathrm{fm} ; \quad \rho_{\mathrm{s}}^{0} \propto \in_{v}=k_{B} T_{c}
$$

Because that -for a formed particle, the cluster of superdense centroids of quasi-electrons are contained (quasi) integrally inside its impenetrable quantum volume $v_{\mathrm{I}}$, we may approximate that - for a protonic $\mathrm{m}$-quark with $\mathrm{N}_{\mathrm{q}} \approx 756$ quasi-electrons with the centroids included in the quark's impenetrable quantum volume of radius $\mathrm{r}_{\mathrm{q}} \approx 0.21 \mathrm{fm}$, we have $\mathrm{d}_{\mathrm{e}}^{\mathrm{m}}\left(\mathrm{T}_{\mathrm{c}}\right) \approx 0,02$ fm at $\mathrm{T}_{\mathrm{c}} \approx \mathrm{T}_{\mathrm{BE}}$, value which gives- by eqn. (21), the approximate value: $\rho_{\mathrm{s}}^{0}\left(\mathrm{~T}_{\mathrm{BE}}\right) \approx 3.15 \times 10^{13} \mathrm{~kg}$, resulting that: $\rho_{\mathrm{s}}\left(\mathrm{a}, \mathrm{T}_{\mathrm{BE}}\right) \approx 2.4 \times 10^{7} \mathrm{~kg}$, value which verify the condition: $\rho_{\mathrm{s}}(\mathrm{a}) / \rho_{\mathrm{s}}(0)<<1$.

The estimated value of the constant $\eta_{\mathrm{v}}$ may be explained by the negentropy introduced by the quantum and subquantum winds of the quantum vacuum, which maintains the integrity of the vortexial structure of the (quasi) electron.

The repulsive force $F_{r s}$ explains -by equations (18) and (21), also the existence of neutral bosons formed as pairs: quark-antiquark or particle-antiparticle, particularly- also the hard gamma quantum transforming into a pair: negatronpositron in a nuclear field, by the conclusion that the $\gamma$ quantum is formed as degenerate pair formed by a negatron and a positron which may be separated and regenerated by a strong electric field.

An argument for the previous conclusions is the fact that a $\left(\mathrm{e}^{-}-\mathrm{e}+\right)$ - pair may be transformed into a neutrino only to an interaction energy higher than those of a hard gammaquantum, $\left(\varepsilon_{\gamma}=2 \mathrm{~m}_{\mathrm{e}} \mathrm{c}^{2}\right)$.
Also, the transforming of a pair $\left(\mathrm{e}^{-}-\mathrm{e}^{+}\right)$into two gamma quanta may be explained by the conclusion of reciprocal charge cancelling. According to the electron model used in CGT, the electric charge is given by the vectorial photons of the electron's surface (vexons, $\mathrm{w}^{ \pm}$) having positive or negative pseudo-charge given by the sense of their spin relative to the electron's magnetic moment and- in consequence, the mechanic interaction between their surfaces permits a $\mathrm{w}^{ \pm}$- photons exchange which cancels the electric charge of both electrons, when are rotates (figure 4).
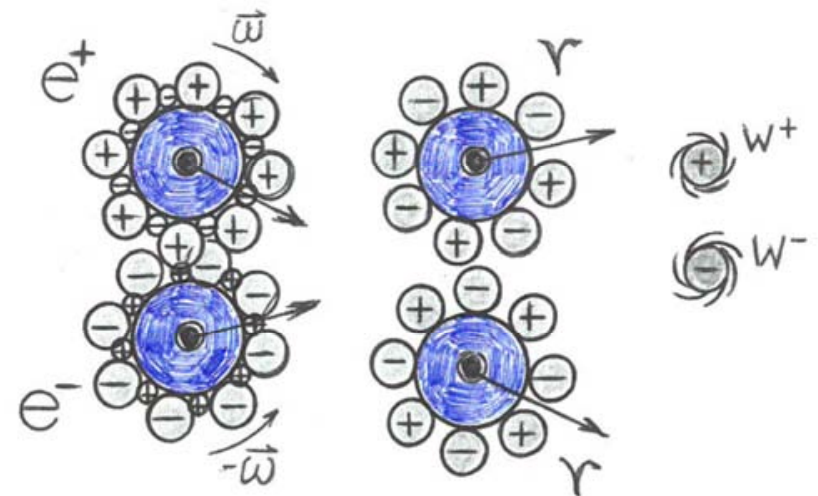

Figure 4. The charges canceling mechanism in the process: $\left(e^{-} e^{+}\right) \rightarrow 2 \gamma$, (CGT). 
It is explained in this way also the fact that- if the interacting electron are not rotated when they collides, they may gives reactions of the form: $\left(\mathrm{e}^{-} \mathrm{e}^{+}\right) \rightarrow\left(\mathrm{e}^{-} \mathrm{e}^{+}\right)$or $\left(\mathrm{e}^{-} \mathrm{e}^{+}\right)$ $\rightarrow\left(\mu^{-} \mu^{+}\right)$.

Because the remanent magnetic moment $\mu_{z}$ of $z^{0}$-preons, given by centrally coupled quasi-electrons, it results thatsimilar to the $z^{0}$-cluster forming case, (fig. 3), is formed initially a quasi-crystallin $z_{2}{ }^{*}$ - pre-cluster, by three $z^{0}-$ preons magnetically and symmetrically attracted by the fourth, i.e. -by the central preon having antiparallel magnetic moment. This $\mathrm{z}_{2}{ }^{*}$-pre-cluster, with three $\mathrm{z}^{0}$-preons with the kernel at the distance $d_{p} \approx a$ from the kernel of the fourth $z^{0}$ preon, generates the $\mathrm{z}_{2}$-zeron cluster of quasi-electronsformed as Bose-Einstein condensate, by the $\mathrm{z}_{2}{ }^{*}$-pre-cluster self-confining- at $\mathrm{T}_{\mathrm{c}} \rightarrow 0 \mathrm{~K}$ or by its confining in a strong magnetic field- at a higher temperature, i.e- by a magnetogravitic force $\mathrm{F}_{\mathrm{MG}}$ generated by the impulse density gradient of the etherono-quantonic vortex-tubes $\xi_{\mathrm{B}}$ which materializes the field lines of the magnetic induction, [2-4].

In specific conditions, without a strong magnetic field but at enough low temperature $\mathrm{T}_{\mathrm{BE}} \geq \mathrm{T}_{\mathrm{c}} \rightarrow 0 \mathrm{~K}$, the $\mathrm{z}_{2}{ }^{*}$-pre-cluster may survive a longer period of time or may attract magnetically three neighboring $z^{0}$-preons, forming a $z_{\pi}^{*}$ pre-cluster of $z^{0}$-preons which generates the $z_{\pi}$-zeron cluster of quasi-electrons, formed as Bose-Einstein condensate by the $z_{\pi}^{*}$-pre-cluster self-confining, (collapsing).

By the loosing of a quasielectron, some $\mathrm{z}_{2}{ }^{*}-$ (pre) clusters becomes $\mathrm{m}_{1}{ }^{+}$-(pre) cluster of quasielectrons, forming a basic $\mathrm{m}_{1}{ }^{+}$- mesonic quark, $\left(\right.$mark $^{+}$- figure $\left.1, \mathrm{a}\right)$ which may be selfconverted into a basic $\mathrm{m}_{2}^{-}$-quark (mark) by the attraction of a negatron $\mathrm{e}^{-}$whose centroid will be incorporated into its quantum volume approximated with radius $r_{\mathrm{z}} \approx \mathrm{a}=1.41 \mathrm{fm}$.

As in the case of the $z^{0}$-cluster, it results that also the $z_{2}-$ zeron and $z_{\pi}$-zeron have a remanent magnetic moment, $\mu_{z^{*}}$ by which they can interact magnetically, forming cold quarks-according to figure 1 , by axially magnetic attraction between them and with $\mathrm{m}_{1}{ }^{+}-$or $\mathrm{m}_{2}^{-}$-basic quark and by the self-confining of the formed $\mathrm{q}^{*}$-pre-cluster, composed of $\mathrm{a}^{ \pm}$ -quark and $z_{\pi^{-}}$and $z_{2}$-zerons. The formed quarks have also a magnetic moment $\mu_{\mathrm{q}}$ by which they can interact magnetically forming initially a quarks pre-cluster which- by magnetic confining in a high magnetic field (comparable with those of a magnetar star: $\mathrm{B} \rightarrow 10^{11} \mathrm{~T}$ ) but lower than a critical value $\mathrm{B}_{\mathrm{c}}{ }^{\mathrm{q}}$, generates an elementary particle, (figure 5).

In consequence, the resulted mechanism of particles and dark bosons cold forming suppose the next steps:

$$
\begin{aligned}
& \text { a) } \mathrm{z}^{0^{*}} \text {-pre-cluster forming } \rightarrow \mathrm{z}^{0} \text { - collapsed cluster (by the aid of magnetic confining); } \\
& \Rightarrow \text { b) } \mathrm{z}_{2}{ }^{*} / \mathrm{z}_{\pi}{ }^{*} \text { - pre-cluster } \rightarrow \mathrm{z}_{2} / \mathrm{z}_{\pi} \text { - cluster (collapsed but without destruction) } \\
& \Rightarrow \text { c) quark/pseudo-quark pre-cluster } \rightarrow \mathrm{q}^{ \pm} / \mathrm{q}^{0} \text { - cluster; }
\end{aligned}
$$

At $\mathrm{T}>\mathrm{T}_{\mathrm{BE}}$, the $\mathrm{z}^{0}$-preon and the $\mathrm{z}_{2}, \mathrm{z}_{\pi}$ bosonic clusters may be still formed in a strong magnetic field which cools the bosonic gas of gammons, generating in the sametime a gravitomagnetic force which increases the density of bosonic gas of quantum vacuum $\gamma^{*}$-gammons and the probability of the Bose-Einstein condensate forming. For a $z^{0}$-cluster forming, i.e- for the bosonic gas of gammons confining, this magnetic field must not exceed a critical value $B_{c}$ equal to the value of magnetic interaction energy between quasielectrons at a distance approximate equal with the reduced Compton wavelenght of the electron: $\mathrm{d}_{\lambda} \approx 386 \mathrm{fm}$ - in accordance to uncertainty relation, resulting that:

$$
\mu_{e} B_{c} \approx \frac{\mu_{0}}{2 \pi} \frac{\mu_{e}^{2}}{d_{\lambda}^{3}} ; \Rightarrow \mathrm{B}_{\mathrm{c}}=\frac{\mu_{0}}{2 \pi} \frac{\mu_{e}}{d_{\lambda}^{3}}=3.23 \times 10^{7} \mathrm{~T}, \mathrm{~d}_{\lambda}=3.86 \times 10^{-13} \mathrm{~m}
$$

Similarly, it results a critical $\mathrm{B}_{\mathrm{c}}$-value also in the case of $\mathrm{z}_{2^{-}}, \mathrm{z}_{\pi^{-}}$or q- cluster forming .

Considering the $\mu^{ \pm}$-lepton having a lifetime: $\tau_{\mu}=2.2 \times 10^{-6}$ sec. as being a single-particle cluster and taking into account that the majority of baryons- considered with $\mathrm{n}=3$ quarks in their sub-structure, have a lifetime: $\tau_{\mathrm{B}} \cong 10^{-10} \mathrm{sec}$. and the

$$
\tau_{k}=\frac{\tau^{0}}{k_{v} \cdot 10^{2 n}} ; \quad \tau^{0} \cong 10^{-14} \mathrm{sec} .
$$

in which: $v_{\mathrm{c}}{ }^{0}$ and $\varepsilon_{\mathrm{c}}{ }^{0}$ represents the critical frequency and the critical phononic energy of particle vibration at which occur not only the proton disintegration but also the quarks disintegration: $\mathrm{T}_{\mathrm{N}} \approx 10^{13} \mathrm{~K},[3,4]$.

In accordance also with the equations (20) and (21), the equation (24) indicates that- at very low temperature: $\mathrm{T} \rightarrow$ majority of mesons $(\mathrm{n}=2)$ have a lifetime $\tau_{\mathrm{m}} \cong 10^{-8} \mathrm{sec}$. at the ordinary temperature: $\mathrm{T} \cong 300 \mathrm{~K}$ of the particles medium, the lifetime of the elementary particles results in CGT inversely proportional to the total intrinsic $\varepsilon_{\mathrm{v}^{-}}$vibration energy of their quarks, according to a semi-empiric relation of approximation:

$k_{v}=\frac{\varepsilon_{v}}{\varepsilon_{v}^{o}}=\frac{n \cdot v_{i}}{v_{c}^{o}}=\frac{n \cdot T}{T_{N}} ; \mathrm{T}_{\mathrm{N}} \cong 10^{13} \mathrm{~K}$

$0 \mathrm{~K}$, the lifetime of the elementary particles increases. In this case, we may deduct that in the cosmic space, especially in the interplanetary and inter-stellary space, may exists also quasi-stable neutral bosons with null spin and with mass comparable with those of the known mesons and baryons, formed by quasi-crystallin neutral pseudo-quarks, i.e.- by 
neutral bosons formed as preonic cluster of $z_{2}$-zerons and $z_{\pi}$ -zerons, which -at $T_{c} \rightarrow 0$, may represents components of cold 'dark matter', as consequence of their (quasi) null emission of electromagnetic radiation and (quasi) null interaction with the electromagnetic radiation.

The basic argument for this consideration is the fact thatrepresenting $\sim 84.5 \%$ from the total matter of the Universe, the ,dark matter' cannot differ very much from the known elementary mesonic and baryonic matter.

Reconsidering the eqns (9) and (10) for cold quarks and cold baryons, in which we replace $\mathrm{m}_{1}^{+}, \mathrm{m}_{2}^{-}$with $\mathrm{z}_{2}$, it results an equation for the mass of possible cold neutral bosons of quantum vacuum, with quasi-crystallin structure of their kernel, formed by neutral pseudo-quarks, in the form:

$$
\mathrm{M}_{\mathrm{Z}}=1_{\mathrm{s}} \cdot \sum_{\mathrm{K}}\left(\mathrm{n}_{1} \cdot \mathrm{z}_{\pi}+\mathrm{n}_{2} \cdot \mathrm{z}_{2}\right) ; \mathrm{z}_{\pi}=7 \mathrm{z}^{0} ; \mathrm{z}_{2}=4 \mathrm{z}^{0} ; \mathrm{n}_{1}=2 \div 5 ; \mathrm{n}_{2} \leq \mathrm{n}_{1} ; \mathrm{K}=1 \div 7,13,19
$$

The limit for $\mathrm{n}_{1}$ is given by the fact that a quasi-crystallin kernel with the length exceeding its diameter $\left(1_{\mathrm{q}}>\mathrm{d}_{\mathrm{q}}\right)$ becomes un-stable because its remanent vibrations: $\epsilon_{\mathrm{v}}=\mathrm{k}_{\mathrm{B}} \mathrm{T}_{\mathrm{c}}$ and the limit for $\mathrm{n}_{2}$ is given by the fact that the preonic 'vacancies' (lacks) generated by the $\mathrm{z}_{2}$-zerons interposed between two $z_{\pi}$-zerons in the continuity of the quasicrystallin network of the boson's kernel gives instability at its remanent vibrations.

The values for $\mathrm{K}$ are given by the fact that the neutral pseudo-quarks: $\mathrm{q}^{0}=\left(\mathrm{n}_{1} \cdot \mathrm{z}_{\pi}+\mathrm{n}_{2} \cdot \mathrm{z}_{2}\right)$ may forms quasi-stable clusters by magnetic radial and axial interactions similary to quarks, which may forms also tetra-, penta-, hexa- or heptaquarks systems, (figure 6).

The values $\mathrm{K}=13 ; 19$, are "magic" numbers for a pseudoquarks cluster, corresponding to a completeness of the quasicrystalline network of the bosonic cluster, (figure 7).

But because that - for $\mathrm{K}=13 \ldots 19$ the formed (super) cluster with the pseudo-quarks $\mathrm{q}^{0}$ coupled radially has the diameter $d_{K}$ greater than its length $l_{K}$, it may results as unstable in interactions with other boson.

A more stable bosonic super-cluster results by a number $1_{\mathrm{S}}$ $>1$ of simple "magic" forms with $\mathrm{K}=13$ or 19 pseudoquarks $\mathrm{q}^{0}$, for which the lenght $\mathrm{l}_{\mathrm{K}}$ of the bosonic super-cluster of cold pseudo-quarks $\mathrm{q}^{0}$ becomes comparable with its diameter: $\mathrm{l}_{\mathrm{K}} \approx \mathrm{d}_{\mathrm{K}}$

As in the case of the pseudo-quark forming, initially is formed a pre-cluster $\mathrm{M}_{\mathrm{z}}{ }^{*}$ of pseudo- quarks $\mathrm{q}^{0}$, which is confined in a super-strong magnetic field, $\xi_{\mathrm{B}}$ - which may explains also the cold genesis of electrons, by dark photons confining around a superdense centroid, resulted by quantons confining in the field of chiral fluctuations of primordial dark energy, according to CGT, [4].

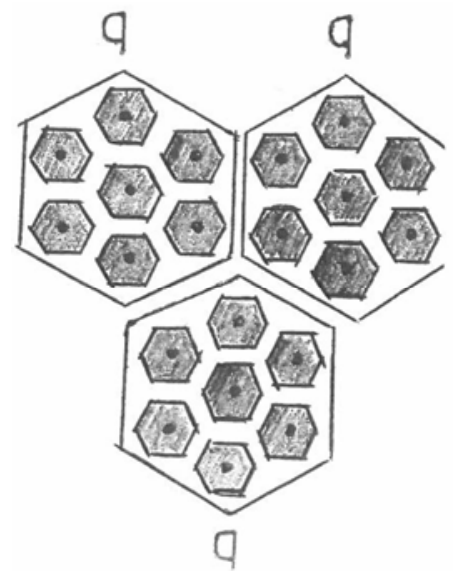

Figure 5. Tri-quark particle.
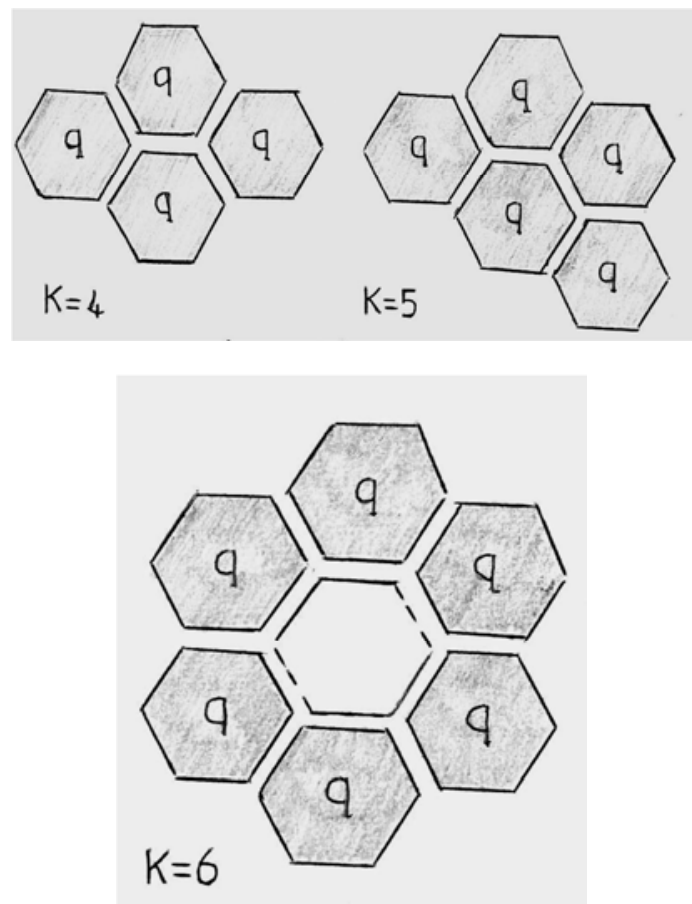

Figure 6. Multi-(pseudo) quark particles.

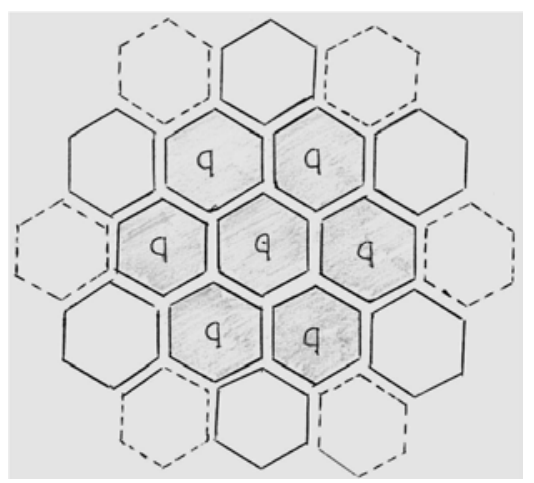

Figure 7. Dark boson with $K \geq 7$ pseudo-quarks

Extrapolating the previous conclusions for the case of cold quarks cluster forming, it results logically the possibility of quasi-cristallin quarks networks forming, considered also for the stelary structures forming, (quark star, [11], [12]), but also the possibility of multi-quark particles forming, (tetra-, penta-, hexa-, hepta-quark particles), possibility which was sustained theoretically and was experimentally confirmed, [13].

By the cold quarks specific to the resulted quasi-crystal model and with the condition $\mathrm{q}=\mathrm{n} \cdot \mathrm{e}$ (integer electric charge), it results as possible some multi-quark particles: 


$$
\begin{aligned}
& \text {-tetra-quark: } \mathrm{P}_{1}^{+}=2 \mathrm{p}^{+}+\overline{\mathrm{m}}_{1}+\bar{\lambda} \approx 2685.4 \mathrm{~m}_{\mathrm{e}} ; 2 \mathrm{p}^{+}+2 \overline{\mathrm{n}^{-}}=2450 \mathrm{~m}_{\mathrm{e}} \\
& \text {-pentaquark: } 4 \mathrm{p}+\overline{\mathrm{m}}_{1}=2580 \mathrm{~m}_{\mathrm{e}} ; 2 \mathrm{p}+2 \lambda+\overline{\mathrm{m}}_{2}=3063.8 \mathrm{~m}_{\mathrm{e}} \\
& \text {-hexa-quark: } 4 \mathrm{p}+2 \mathrm{~m}_{2}=2720.4 \mathrm{~m}_{\mathrm{e}} ; 3 \mathrm{p}^{+}+3 \mathrm{n}^{-}=3675 \mathrm{~m}_{\mathrm{e}} ; \\
& \text {-hepta-quark: } 4 \mathrm{p}+2 \overline{\mathrm{m}}_{1}+\mathrm{n}=3329 \mathrm{~m}_{\mathrm{e}} ; 4 \mathrm{p}+\overline{\mathrm{p}}+\mathrm{n}+\overline{\mathrm{n}}=4323.6 \mathrm{~m}_{\mathrm{e}} ; 4 \mathrm{p}+\mathrm{n}+2 \bar{\lambda}=4762.2 \mathrm{~m}_{\mathrm{e}}
\end{aligned}
$$

With a smaller probability there are possible theoretically also quarks, pseudo-quarks, neutral bosons and charged particles with preonic quasi-crystalline structure based on a triangular form of the $\mathrm{z}^{0}$-preon, (figure 3 ), with the resulted bosonic mass in the form:

$$
\mathrm{M}_{\mathrm{Z}}=\sum_{\mathrm{K}}\left(\mathrm{n}_{1} \cdot 6 \mathrm{z}^{0}+\mathrm{n}_{2} \cdot 3 \mathrm{z}^{0}\right) \text {, with: } \mathrm{z}_{\pi}=7 \mathrm{z}^{0} ; \mathrm{z}_{2}=4 \mathrm{z}^{0} ; \mathrm{K}=1 \div 7 ; \mathrm{n}_{1}=1 \div 4 ; \mathrm{n}_{2} \leq \mathrm{n}_{1} .
$$

The generating of particles with bigger mass than those of particles that enters in reaction may be explained in CGT, according to the previous conclusions, by the decomposing of quantum vacuum zerons and of other neutral bosons formed as quasi-crystallin cluster $\mathrm{M}_{\mathrm{z}}\left(\mathrm{m}_{\mathrm{z}}\right)$ of $\mathrm{z}^{0}$-preons, into real ( $\mathrm{q}-\mathrm{q})$-pairs, by the $\mathrm{Q}_{\mathrm{i}}$ - interaction energy, when $\mathrm{Q}_{\mathrm{i}} \approx \mathrm{E}_{\mathrm{q}}$ $=\mathrm{m}_{\mathrm{z}} \mathrm{c}^{2}$.

This theoretical possibility, resulted physically as consequence of the resulted quasi-crystallin form of the quark, may explain the possibility of heavy and ultra-heavy particles forming, with mass over $4 \mathrm{GeV} / \mathrm{c}^{2}$, but because that the increasing of the quark's length gives a higher instability to the quarks cluster, the particles formed with heavy and ultra- heavy quarks are more un-stable and are identifiable experimentally as "resonances", such as: the scalar meson $\mathrm{a}_{1}$ $\left(2465.7 \mathrm{~m}_{\mathrm{e}}\right)$, the charmed Sigma: $\Sigma_{\mathrm{c}}{ }^{*}\left(4929 \mathrm{~m}_{\mathrm{e}}\right)$ and so on. This fact may be important also for the case of the transition of baryonic matter to quark matter, which is supposed to occur inside the neutron star core when the matter density exceed the neutron's density, [11, 12], being sustained also the conclusion that the accreting X-ray binary pulsars may be strange stars that are composed of color-flavor locked quark matter throughout [14] and the hypothesis that a possible crystalline phase of quark matter may explain the "glitch" of the neutron star observed as pulsar [12], consisting in the increase of the angular velocity at the crust of the star.

According to the proposed quasi-crystalline quark model, inside a rotational neutron star with an enough strong magnetic field (magnetar) and with a mass which allows its transforming into 'black hole', it is possible the forming also of super-quarks, corresponding- in eqn. (9), to $k \geq 5$, by the increasing of the matter density until the density of the current mass of the neutron quarks, $(u, d)$, i.e: $\rho_{\mathrm{n}}{ }^{0} \approx 5 \times 10^{17}$ $\mathrm{kg} / \mathrm{m}^{3},[1-4]$.

The "hot" equivalent of this process is -according to CGT, the forming of heavier quarks and particles from strong interaction between lighter quarks and particles with the contribution of quarks resulted from bosons $\mathrm{M}_{\mathrm{b}}\left(\mathrm{m}_{\mathrm{b}}\right)$ of the polarized quantum vacuum, when the interaction energy, $\mathrm{Q}_{\mathrm{i}}$, is at least equal with the intrinsic energy $m_{b} c^{2}$ of the "splitted" boson and allow the transforming of its 'virtual' quarks in real (stable individualized) quarks, like in the reaction:

$$
\begin{gathered}
\text { (Exp.) } \pi^{-}\left(\overline{\mathrm{m}}_{1}+\mathrm{m}_{2}\right)+\mathrm{p} \mathrm{r}\left(2 \mathrm{p}^{+}+\mathrm{n}^{-}\right)+\mathrm{Q} \rightarrow \Lambda^{\mathrm{o}}(\mathrm{s}+\mathrm{n}+\mathrm{p})+\mathrm{K}^{\mathrm{o}}\left(\mathrm{m}_{2}+\bar{\lambda}\right) \\
\overline{\mathrm{m}}_{1}+\mathrm{p}^{+}+\mathrm{Q} \rightarrow \overline{\mathrm{m}}_{1}+\mathrm{m}_{1}+2 \mathrm{z}_{\pi}+\mathrm{Q}=\mathrm{z}_{2}+2 \mathrm{z}_{\pi}+\mathrm{Q} \rightarrow\left(\mathrm{s}^{-}+\overline{\mathrm{s}} ; \overline{\mathrm{s}}^{+} \rightarrow \bar{\lambda}+\mathrm{z}_{2} ;\right. \\
\mathrm{s}^{-}+\mathrm{n}^{-}+\mathrm{p}^{+} \rightarrow \Lambda^{\mathrm{o}} ; \bar{\lambda}^{+}+\mathrm{m}_{2} \rightarrow \mathrm{K}^{\mathrm{o}} ; \text { - (permitted reaction). }
\end{gathered}
$$

According to the previous theoretical conclusion it is possible that some massive bosons experimentally evidenced at very high energies, of few $\mathrm{TeV}$, such as the Higgs boson, are produced as particulary cases, by the reaction energy and the rest-mass energy of other lighter bosons of the quantum vacuum, with higher probability in zones with high magnetic field as those of a magnetar, $\left(\mathrm{B} \rightarrow 10^{12} \mathrm{~T}\right)$ which may generates also dark photons and gammons (pairs of electrons, $\left.\mathrm{e}^{+}-\mathrm{e}^{-}\right)$, according to CGT [4].

\section{Conclusions}

By a preonic quasi-crystalline quark model, of BoseEinstein gammons condensate type and by a cold genesis theory of matter and fields, which predicted the existence of a preon $\mathrm{z}_{0} \approx 34 \mathrm{~m}_{\mathrm{e}}$, experimentally evidenced in 2015 , it is explained the elementary particles cold genesis by two preonic cold genesis bosons with hexagonal symmetry: $\mathrm{z}_{\pi}=$ $7 z^{0} ; z_{2}=4 z^{0}$, by a mechanism with the next steps:

a) $\mathrm{z}^{0^{*}} / \mathrm{z}^{0}$-pre-cluster/cluster forming (by magnetic confining);

b) $z_{2}{ }^{*} / z_{2}$ - and $z_{\pi}^{*} / z_{\pi^{-}}$(collapsed) pre-cluster/cluster forming;

c) $\left(\mathrm{q}^{ \pm} / \mathrm{q}^{0}\right)$ - quark or pseudo-quark pre-cluster/cluster forming;

d) pre-cluster of quarks or pseudo-quarks forming;

e) elementary particle or dark boson forming.

The previous possibility sustain the theory of the elementary particles cold genesis with the aid of a very strong magnetic field, of a magnetar or a gravistar, (CGT, [14]) by the forming of quasi-crystalline preonic quarks and particles, as Bose-Einstein condensate of $\mathrm{N}$ gammons, considered as pairs $\left(\mathrm{e}^{*_{+}}-\mathrm{e}^{*_{-}}\right)$of quasi-electrons with diminished mass $\mathrm{m}_{\mathrm{e}}{ }^{*}$, charge $\mathrm{e}^{*}$ and magnetic moment $\mu_{\mathrm{e}}{ }^{*}$, in the field of an etherono-quantonic vortex of the magnetic 
moment: $\Gamma_{\mu}^{*}(\mathrm{r})=\Gamma_{\mathrm{A}}+\Gamma_{\mathrm{B}}$, formed of sinergonic etherons $\left(\mathrm{m}_{\mathrm{s}}\right.$ $\approx 10^{-60} \mathrm{~kg}$ )- generating the magnetic potential $\mathrm{A}$ and of quantons $\left(\mathrm{m}_{\mathrm{h}}=\mathrm{h} \cdot 1 / \mathrm{c}^{2}=7.37 \times 10^{-51} \mathrm{~kg}\right)$ - generating vortextubes $\xi_{\mathrm{B}}$ that materializes the field lines of magnetic induction $\mathrm{B}$.

The resulted preonic quark model predicted the existence of a preon $\mathrm{z}_{0} \approx 34 \mathrm{~m}_{\mathrm{e}}$, experimentally evidenced in 2015 but considered as $\mathrm{X}$ - boson of a fifth force, of leptons to quark binding.

According to the model, the stability of quarks is explained by a quasi-crystalline model of $z^{0}$-preon and of the quark core, which results by the pre-cluster's cold collapsing.

By this quasi-crystalline preonic quark model, with hexagonal symmetry, there are identified as possible some dark matter constituents, resulted as bosons of quantum vacuum with null charge, (quasi) null magnetic moment and with stability comparable to those of particles from the cosmic radiation, resulted by the kernel's crystallinity with hexagonal or triangular symmetry of the $z^{0}$-preon, with masses corresponding to the relations:

$$
\begin{gathered}
M_{Z}=\sum K\left(n_{1} \cdot z_{\pi}+n_{2} \cdot z_{2}\right) ;\left(\text { with hexagonal } z^{0} \text {-preon); } M_{Z}=\sum K\left(n_{1} \cdot 6 z^{0}+n_{2} \cdot 3 z^{0}\right) \text {, (with trigonal } z^{0}\right. \text {-preon) with: } \\
\mathrm{z}_{\pi}=7 \mathrm{z}^{0} ; \mathrm{z}_{2}=4 \mathrm{z}^{0} ; \mathrm{K}=1 \div 7 ; \mathrm{n}_{1}=1 \div 4 ; \mathrm{n}_{2} \leq \mathrm{n}_{1} .
\end{gathered}
$$

It is sustained also- according to the explicative model, the conclusion that -being of more than $80 \%$ from the total matter of the Universe, the dark matter is not very different from the ordinary matter.

It results also some predictions for cold multi-quark particles (of cold genesis), such as:

$2450 \mathrm{~m}_{\mathrm{e}} ; 2685.4 \mathrm{~m}_{\mathrm{e}}$ tetra-quark; $3063.8 \mathrm{~m}_{\mathrm{e}}$ penta-quark;

$2720 \mathrm{~m}_{\mathrm{e}} ; 3672.4 \mathrm{~m}_{\mathrm{e}}$ hexa-quark;

$3329 \mathrm{~m}_{\mathrm{e}} ; 4762.2 \mathrm{~m}_{\mathrm{e}}$ hepta-quark.

The chirality of the electron's centroid, of radius $\sim 10^{-18} \mathrm{~m}$, generates the e-charge's sign, by a supposed helicoidal form which may explain also the electron's helicity (the parallel/antiparallel orientation of the spin related to the particle's impulse)-according to CGT.

The proposed particle model corresponds to an organized (quasi-crystallin) collapsed Bose-Einstein Condensate, but with quasicrystallin kernel, formed by electronic centroids.

It results-according to the model, that the neutronic stars emitting gamma-rays and with strong and variable magnetic field, of magnetar type, may create conditions for cold particles production, as collapsed BEC with quasi-crystallin kernel.

Also, it results that a process in which an electron and a positron annihilate, producing a photon that becomes a quarkantiquark pair generating thereafter a gluon, (process considered by the quantum mechanics), cannot occur without the participation of real bosons of the quantum vacuum, with organized (quasi-crystallin) kernel, which are formed as real pairs: quark-antiquark.

Also, the forming of "quark stars" as quasi-crystalline network of quarks, supposed in the case of Cygnus X3 star [15], results- by the theoretic model of quasi-crystalline quarks genesis, as plausible possibility by the transforming of a magnetar into a rotational 'black hole' with electric charge on surface, which may generates an enough strong magnetic field for diminish the repulsive scalar charge of quarks (given by Brownian energy of quantonic clusters, conform to CGT [16]) by the dynamic quantum pressure of the etheronoquantonic vortex-tubes $\xi_{\mathrm{B}}$ of magnetic field lines.

In the same time, this possibility sustains- by the proposed model of particles forming, the possibility of dark super-heavy particles/bosons existence- supposed by some dark matter models, by the hypothesis of massive quarks/pseudo-quarks cold forming, in a strong magnetic field of neutronic or "black hole" stars (magnetaric field) by nucleons cluster collapsing, with the maintaining of the quasicrystallin arrangement at the new formed particle'/boson's level, as consequence of the diminishing of the scalar repulsive charge of the nucleonic impenetrable quantum volume (of $r_{i} \approx 0.45 \div 0.6 \mathrm{fm}$ ) by the super-strong magnetic field.

In a Gross-Pitaevskii equation, of the BEC's wave function, this possibility can be equated by considering a negative value of the coherent scattering lengt (a) -as consequence of the magnetic field's action, (depending on the magnetic B-field), which may transforms the repulsion between the nucleonic impenetrable quantum volumes into an attractive interaction, the external potential $\mathrm{V}(\mathrm{r})$ being- in this case, a magnetogravitic potential $\mathrm{V}_{\mathrm{MG}}$ given by the local gradient $\nabla_{\mathrm{r}}\left(\rho_{\Gamma} \mathrm{c}\right)$ of the etherono-quantonic vortex-tubes $\xi_{\mathrm{B}}$ that materializes the field lines of magnetic induction B, [1-4].

It is known that was considered theoretically also the possibility to describe the dark matter as a non-relativistic, Newtonian Bose-Einstein gravitational condensate, [17] but at the atomic level.

The resulted explicative model of particles cold genesis may explain the existence of a huge number of material particles in the Universe by the conclusion of cold ("dark") photons and thereafter- of electronic neutrinos and cold electrons genesis in the Cold Proto-Universe's period, by chiral (vortexial) fluctuations in the 'primordial dark energy' (p.d.e.)-considered in CGT as omnidirectional fluxes of etherons and quantons circulated through a brownian part of etherons and quantons, at densities of p.d.e. comparable with those of the E-field quanta at the electron's surface $\left(5.17 \times 10^{13} \mathrm{~kg} / \mathrm{m}^{3}\right.$, according to CGT $[3,4]$, specific to a magnetaric magnetic field, by the relation: $\mathrm{B}=\mathrm{E} / \mathrm{c}$ ).

These chiral fluctuation of p.d.e., could confine quantons, forming quantonic clusters which became centroids of vectorial photons and of electrons, ensuring the particle's stability, the cold pseudo-scalar photons resulting in CGT as pairs of vectorial photons, excepting the case of hard gammaquanta which represents pairs of degenerate electrons with opposed charges, according CGT, [3, 4, 9].

These conclusions are sustained also by the revised model of photon [9] and by the composite fermion model of electron resulted in CGT, [1-4], in accordance with the possibility of the energy-to-matter conversion, resulted from 
the relation: $\mathrm{E}=\mathrm{mc}^{2}$.

The fact that CGT -based on a galileian relativity, deduces a non-null rest-mass of photon: $\mathrm{m}_{\mathrm{f}}{ }^{0}=1 / 2 \mathrm{hv} / \mathrm{c}^{2}$, which contributes to the total mass of the electron as photons of its quantum volume, but considering also the existence of an etherono-quantonic zero-point energy with brownian kinetics which increases the total density of the quantum vacuum, is not contradictory because that the etherono-quantonic medium is a super-fluid with extreme low viscosity, whichaccording to d'Alembert paradoxe [18], generates a cvasinull drag force against the moving photon.

For coupled photons in antiphase (with four vectorial photons), the resulted quanta are of scalar radiation and in the domain of microwaves may appear as axion.

According to CGT, it results-in consequence that the vacuum fluctuations, supposed by P.I.Fomin (1970, 1975) and E.P.Tyron (1973, [19]) as possible mechanism of particle-antiparticle pairs production, without sustainable explanations , may explain the Universe matter's cold genesis but only as chiral, vortexial fluctuations which are the real equivalent of the used concept of "virtual particles" and which generates primordial dark energy- to matter conversion by quantons confining, with the generation of stable quantum simple and composite leptonic fermions and thereafter- of bosons and elementary particles (mesons and baryons).

The previous conclusions sustains also the possibility of a real increase of the particle's mass, charge and magnetic moment, in a strong magnetic field, by an supplementary etherono-quantonic vortex $\Gamma_{\mathrm{r}}(\mathrm{r})$ which is added to the similar vortex $\Gamma_{\mu}(\mathrm{r})$ of the particle's magnetic moment by the vortextubes $\xi_{\mathrm{B}}$ of the magnetic field, which increased the particles magnetic moment: $\mu_{\mathrm{p}} \sim \Gamma_{\mathrm{T}}(\mathrm{r})=\Gamma_{\mathrm{r}}(\mathrm{r})+\Gamma_{\mu}(\mathrm{r})$ and the magnetogravitic potential $\mathrm{V}_{\mathrm{MG}}(\mathrm{r})$ generated by $\Gamma_{\mathrm{T}}(\mathrm{r})$, but until a limited value depending on the magnetic B-field's value.

\section{References}

[1] Arghirescu, M., "The material structures genesis and field effects", Ed. MatrixROM, Bucharest, 2006.

[2] Arghirescu, M., "The Cold Genesis", Ed. Invel- Multimedia, 2011; viXra, 1104.0043, 2012.

[3] Arghirescu, M., "The Cold Genesis of Matter and Fields", Ed. SciencePG, 2015.

[4] Arghirescu, M., 'A Quasi-Unitary Pre-Quantum Theory of Particles and Fields and SomeTheoretical Implications', IJHEP, July, 80-103, 2015.
[5] Arghirescu, M., 'A preonic quasi-crystal quark model based on a cold genesis theory and on the experimentally evidenced neutral boson of $34 \mathrm{~m}_{\mathrm{e}}$ ', Global Journal of Physics Vol. 5, No 1, 2016, pp. 496-504.

[6] Arghirescu, M., "The Experimental Evidences for a $34 \mathrm{~m}_{\mathrm{e}}$ Neutral Boson, Predicted by a Particles Cold Genesis Theory, as Argument for a Preonic Quark Model", IJHEP, Vol. 3, No. 4, 2016, pp. 25-32.

[7] Islama, M. M. \& Luddyb, R. J., "High Energy Elastic Scattering in Condensate Enclosed Chiral Bag Model and TOTEM Elastic Measurements at LHC at 7TeV", The EDS Blois, 9-13 sept. 2013.

[8] Yan, Y., \& Tegen, R., "N-N Scattering and Nucleon Quark core", Science Asia, 27, 251, 2001.

[9] Arghirescu, M., "A Revised Model of Photon Resulted by an Etherono-QuantonicTheory of Fields", Open Access Library Journal, 2: e1920, 2015.

[10] R. D. Chipman, L. D. Jennings, Phys. Rev. 132 (1995) 728; Greenberg, O. W., Yodh, G. B., Phys. Rev. Lett., 32,1974, p. 1473 .

[11] H. Chen, G. F. Burgio, H. J. Schulze, N. Yasutake, 'Structure of the hadron-quark mixed phase in protoneutron stars', Astronomy \& Astrophysics Journ., Vol. 551, March 2013, A13.

[12] M. G. Alford, K. Rajagopal et al., ,Color Superconductivity in dense Quark Matter', arXiv:0709.4635v2, 2008.

[13] Aaij, R. et al. (LHCb collaboration) "Observation of J/ $\psi p$ resonances consistent with pentaquark states in $\Lambda_{b}^{0} \rightarrow J / \psi K \mathrm{~K} p$ decays", Physical Review Letters, 115 (7), 2015; arXiv: 1507.03414.

[14] Madsen, J., Phys. Rev. Lett. 85, 10, 2000.

[15] Merali, Z., 'Astronomers look to quark stars for a fifth dimension', New Scientist, 23 June 2007.

[16] Arghirescu. M., 'A Correspondence with the Bag Model of a Pre-quantum B.-E. Condensate Model of Nucleon', IJHEP, Vol. 3, Issue 2, April, 10-17, 2016.

[17] Böhmer, C. G., Harko, T., 'Can dark matter be a BoseEinstein condensate?'; arXiv:0705.4158v4 [astro-ph] 21 Jun 2007.

[18] Saint-Venant, A. 'Mémoire sur la théorie de la résistance des fluides. Solution du paradoxe proposé à ce sujet par d'Alembert aux géomètres. Comparaison de la théorie aux expériences", Comptes Rendus des Séances de l'Académie des Sciences, 24: 243-246, 1847.

[19] Tyron, E.P., "Is the Universe a Vacuum Fluctuation?" Nature vol. 246,1973 , p.p. 396-397. 